\title{
Pelvic pain rehabilitation
}

\author{
Meltem Vural(1) \\ Department of Physical Medicine and Rehabilitation, University of Health Sciences, Bakırköy Dr. Sadi Konuk Training and Research Hospital, İstanbul, Turkey
}

Received: August 13, 2018 Accepted: September 12, 2018 Published online: November 04, 2018

\begin{abstract}
Chronic pelvic pain is defined as persistent painful condition which lasts for at least six months under umbilicus. Numerous factors are blamed for etiopathogenesis, and quality of life of individuals is adversely affected. Chronic pain as well as functional disorders are accompanied to chronic pelvic pain. The treatment and rehabilitation program should be tailored for specific causes, targeting general pain treatment. Consequently, chronic pelvic pain management can be used to propose personalized treatment options and include patient education, behavioral therapy, and a biopsychosocial approach. Interdisciplinary teamwork and collaboration are essential for facilitating patient-centered rehabilitation.
\end{abstract}

Keywords: Pelvic floor; pelvic pain; pelvic rehabilitation; physical therapy.

Pelvic pain is defined as a painful condition located pelvis, lumbosacral region, pelvic floor, and the anterior abdominal wall at or below the umbilicus. ${ }^{[1]}$ On the other hand, chronic pelvic pain (CPP) is a syndrome of pain originating from a specific site of the body region for at least six months, resulting in functional disability or severe condition requiring treatment. It may be caused by one or more different conditions and seriously affects the individual's work, family, and social life. ${ }^{[2,3]}$ It is expressed as an increase in somatic pain sensitivity in the extragenital region; it is not known whether there are comparable differences in the pelvic floor and genitourinary pain sensitivity. It is a serious problem in the clinical practice that its etiology has not been fully understood yet, and it has a complex, multifactorial natural history and is resistant to treatment. ${ }^{[4]}$ It is reported that $25 \%$ of women and 2 to $10 \%$ of men are affected by CPP. ${ }^{[5]}$ In the United States (US), it accounts for about $10 \%$ of the admissions to urogynecologists with a varying prevalence of 5.7 to $26.6 \%{ }^{[4]}$ In addition, CPP is one of the causes of more than $\$ 800$ million cost per year in the health care system. ${ }^{[6]}$ In addition to pain symptoms, bladder or bowel dysfunction, sexual dysfunction, systemic or constitutional symptoms, depression and anxiety disorders can be seen in these patients. Consequently, quality of life of patients is seriously and adversely affected. ${ }^{[7]}$

\section{ETIOLOGY}

Due to its complex etiopathogenesis and different anatomical structures, CPP needs to be examined systematically and in a sexually-specific way. On the other hand, even with experienced clinicians, the diagnosis challenges are occasionally encountered. Interdisciplinary evaluation of patients is, therefore, important. ${ }^{[8]}$ The common causes of CPP are shown in Table 1. As described in many pain states, CPP is also associated with central changes. The changes in the behavioral and central response of the harmful stimulation are confronted as the altered activity of both the hypothalamic-pituitary-adrenal axis and of the autonomic nervous system, as a central pain. It has been shown that psychological distress also contributes. ${ }^{[9]}$ Different psychosocial factors and psychiatric disorders such as increased pain sensitivity, stress, personality characteristics, social aspects of individuals, depression, anxiety, and trauma related disorders, somatization, and substance abuse have

Corresponding author: Meltem Vural, MD. SBÜ, Bakırköy Dr. Sadi Konuk Eğitim ve Araştırma Hastanesi Fiziksel Tıp ve Rehabilitasyon Kliniği, 34147 Bakırköy, İstanbul, Turkey. e-mail: drmeltemvural@gmail.com 
been also found to be associated with CPP. ${ }^{[10]}$ Therefore, psychosocial factors should also be considered in these patients.

\section{EVALUATION, DIAGNOSIS, AND DIFFERENTIAL DIAGNOSIS OF CPP}

A careful and detailed history is extremely important for CPP. The history of the patient with CPP should include urinary, gastrointestinal, gynecological, musculoskeletal, sexual, and psychosocial symptoms. ${ }^{[5]}$ Specific features of pelvic pain, location, duration, reflected pain, specific movements that increase pain, and specific positions including prolonged sitting, sexual intercourse, and exercises should be questioned. Whether it is accompanied by neuropathic pain, pelvic infection history, symptoms of urinary system, frequency of urine, urgency, incontinence, noxiousness, dizziness, feeling of complete voidance of the bladder and painful bladder should be investigated. ${ }^{[7]}$ It is recommended that the severity of the pain should be assessed through special assessment tools, particularly the Visual Analog Scale and its digital versions. ${ }^{[3]}$ In addition, in recent years, defining pain on the anatomical areas on a chart of pain has been increasingly used as a method of confrontation.

Table 1. Common causes of chronic pelvic pain

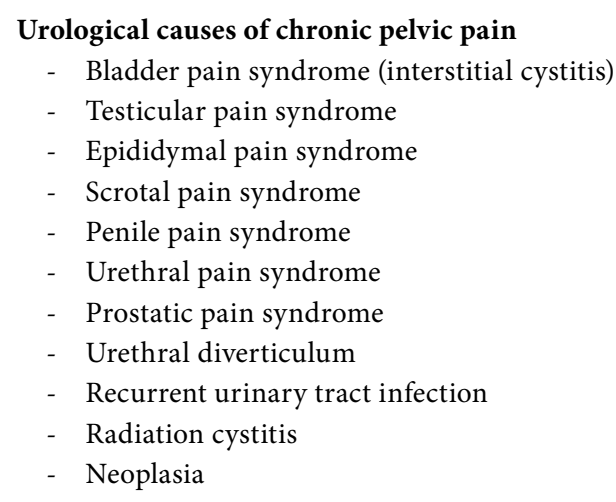

Urogynecological causes of chronic pelvic pain

- Endometriosis

- Adenomyosis and uterine fibroids

- Chronic pelvic inflammatory disease

- Pelvic adhesions

- Pelvic congestion syndrome

- Residual ovary syndrome

- Leiomyoma

- Infectious salpingitis

- Benign cystic mesothelioma

- Postoperative peritoneal cysts

- Neoplasia

\section{Gastrointestinal causes of chronic pelvic pain}

- Irritable bowel syndrome

- Inflammatory bowel disease and other causes of colitis

- Diverticular disease

- Chronic intermittent bowel obstruction

- Neoplasia

- Chronic constipation

- Celiac disease

- Functional abdominal pain syndrome

- Opioid-induced bowel dysfunction

- Hemorrhoids

- Anal fissures

- Perineal pain syndrome

\section{Musculoskeletal disorders}

- Myofascial pain syndrome (Piriformis, levator ani, iliopsoas, obturator internus and quadratus lumborum muscles)

- Coccydynia

- Piriformis syndrome

- Hip osteoarthritis

- Sacroiliac joint dysfunction

- Pelvic insufficiency fractures

- Osteitis pubis

- Fibromyalgia

- Spinal lesions (Degenerative disk disease, herniated nucleus pulposus, facet joint disorders, infections and neoplasia)

\section{Neurological disorders}

- Plexopathy

- Radiculopathy

- Nerve entrapment syndrome (Pudendal, obturator, iliohypogastric, ilioinguinal, genitofemoral and lateral femoral cutaneous and anterior abdominal cutaneous nerves)

- Chronic pain syndrome

- Abdominal epilepsy

- Abdominal migraine

\section{Psychosocial causes of chronic pelvic pain}

- Somatization

- Depression

- Anxiety

- Substance and alcohol abuse

- Physical and sexual abuse

- Sexual dysfunction

- Family or relationship problems 
Furthermore, a detailed physical examination, diagnostic tests, and evaluation for specific causes should be performed. ${ }^{[11]}$ Physical examination should include evaluation of the patient's posture, gait, body, abdomen, lumbar region, hip joint, thigh muscle, and connective tissues as well as bone, pelvic floor muscles and ligamentous structures in the pelvic region. Sensitive and trigger points and reflected pain should be defined, and a thorough neurological examination should be performed.

The interdisciplinary approach is extremely important in the diagnosis and treatment of CPP and this issue has been particularly emphasized in recent studies. ${ }^{[12,13]}$ In the assessment of pain, the skin and subcutaneous tissue should be checked by gently rolling with the first two fingers. Sensitive areas should be questioned for thickening, tension, sharp pain, and feeling of pain. By compressing the bladder, pressure is applied to the suprapubic area to assess whether pain is present. The presence of a scar tissue should be evaluated. Palpation, pelvic floor muscle tone, and trigger points should be also examined. Lumbar region, abdomen, pelvis, and thigh muscles should be examined for taut band and trigger points. ${ }^{[1]}$ Pelvic myofascial pain is particularly common in the levator ani, hip internal rotators (particularly obturator internus), and connective tissue. Chronic prostatitis in men, interstitial cystitis/bladder pain syndrome in women and men, and vulvodynia in women may also lead to CPP. About 60 to $85 \%$ of patients with CPP have trigger points in the levator ani and obturator internus muscles. ${ }^{[14,15]}$ In a study, $92 \%$ of women with myofascial pelvic pain syndrome had a trigger point in the levator ani, $45 \%$ in the obturator internus, $43 \%$ in the iliopsoas, and $8 \%$ in the piriformis muscles. ${ }^{[16]}$ It should be kept in mind that myofascial pelvic pain may be responsible for CPP and may be related to symptom diversity. Physical examination methods for the diagnosis of myofascial pelvic pain and for evaluating the pelvic muscle system have changed considerably and are not often defined. In CPP, considering the relationship between the known role of myofascial pelvic pain and the symptoms of lower urinary tract symptoms, further studies should be conducted to increase the awareness of physicians in this area. Furthermore, the development and standardization of reliable and reproducible examination methods is required. ${ }^{[1]]}$

The Carnett's sign and cotton swab testing are also helpful, when the pain is caused by trigger points at the abdominal wall, or when evaluating abdominal wall neuropathy. The Carnett's sign is a clinical examination finding that is useful to confirm whether pain originates from the abdominal wall. To perform a test for the Carnett's sign, the clinician should ask the supine patient to lift his/her head off the bed. A positive Carnett's sign is identified, when the pain worsens during the test. Allodynia or hyperalgesia is assessed by a cotton swab test (Figure 1). The cotton swab test is also used to assess vulvar pain (vulvodynia) or women with dyspareunia. The cotton swab is moved medially from the side of the thigh to move slightly to the vestibular area. Clitoral and anal reflex should be also tested. ${ }^{[17,18]}$ The internal pelvic floor, the obturator internus muscle and the pudendal nerve are assessed for irritation by intravaginally palpating in the direction of 3 and 9 o'clock. Then, the urogenital diaphragm and levator ani muscle groups are examined in turn. The pubococcygeus muscle is palpated in the direction between 7 and 11 o'clock on the left and 1 and 5 o'clock on the right, and the puborectalis is deeper and palpated parallel to the ground in the direction of 6 o'clock. The coccygeus muscle is estimated between in the direction of 4 and 8 o'clock. In addition, it should be questioned whether patients should do the Kegel exercises and whether there is pain during the exercises. ${ }^{[17]}$

In CPP, biochemical and hormonal tests should be requested according to the history and physical

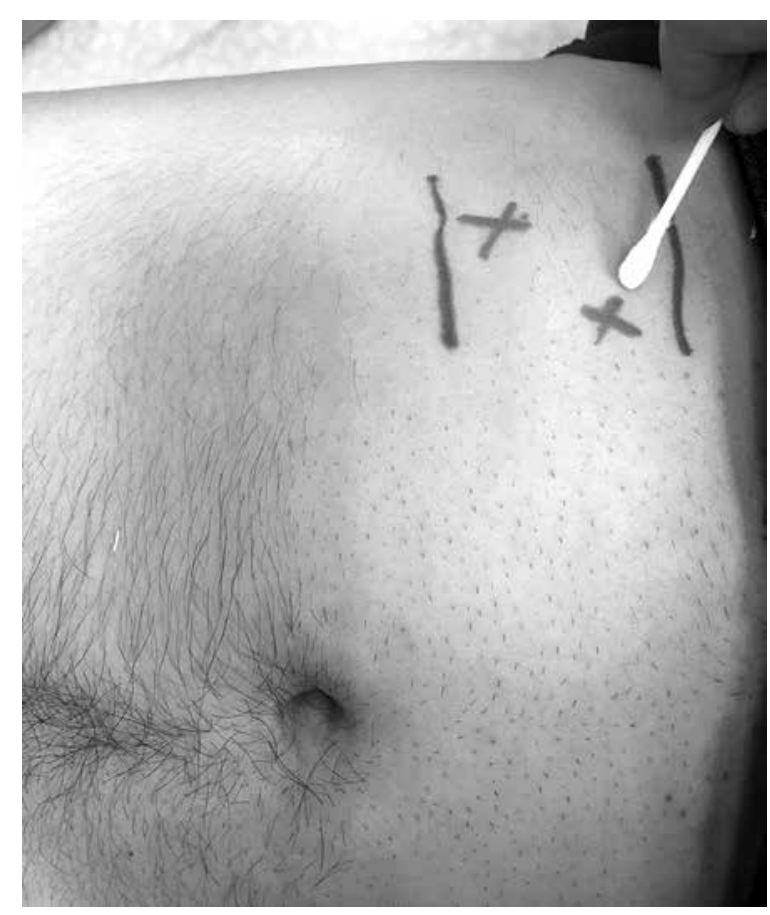

Figure 1. Cotton swab test. Cotton swab can be used to assess allodynia or hyperalgesia, suggesting a possible abdominal wall neuropathy. 
examination findings of the patients. If necessary, complete urinalysis, complete blood count, pregnancy test, Chlamydia and Gonorrhea, culture studies for Trichomonas infections should be done. The travel history is valuable in terms of exclusion of intestinal infections caused by gastrointestinal symptoms. Imaging methods such as direct radiography, diagnostic ultrasonography (USG), computed tomography (CT), magnetic resonance imaging (MRI), and MRI neurography can be used. ${ }^{[19]}$ The MRI neurography for sciatic, femoral, obturator, lateral femoral cutaneous, genitofemoral, ilioinguinal, iliohypogastric, pudendal, and posterior femoral cutaneous nerves are available to evaluate the common pathological conditions of large and small pelvic nerves. ${ }^{[6]}$ In addition, cystoscopy and laparoscopy are among the diagnostic methods used in patients with CPP. ${ }^{[20,21]}$ A case diagnosed by USG and presented with anterior abdominal wall endometriosis is shown in Figure 2.

The concepts of groin pain and pelvic pain often cause confusion. Groin pain is commonly observed among active adults. Complex anatomy and biomechanical properties make it difficult to diagnose by clinicians. It is indispensable to evaluate the hip effectively, to perform a good physical examination in understanding the local anatomy and function. ${ }^{[22]}$ The groin pain may be due to the pathology of the hip joint; spinal pathologies, and inguinal hernias, and can also be confounded by extra-articular causes. In addition, the reflections of the pain of other organs and systems may show itself in the groin area. ${ }^{[12]}$ In intra-articular pathologies, pain is mostly encountered as groin or inguinal pain. Patients often localize pain on their biceps in a certain way. Pain in the hip joint with internal rotation on physical examination is sensitive and specific to the hip pathology. Intra-articular local anesthetic injections and subsequent provocative tests are often used for the differential diagnosis. ${ }^{[23-25]}$ Symptoms, such as neurogenic claudication and whether symptoms increase with coughing at the distinction of spinal pathologies, should be questioned. A positive straight leg raise test, femoral stretch test, reduction of reflexes, sensory loss or decrease, decrease in the muscle strength and muscle atrophy should be also investigated. When necessary, epidural injections are helpful to identify the source of pain and to decide the treatment strategy. ${ }^{[23]}$ Causes of groin pain are shown in the Table 2.

\section{CHRONIC ABDOMINAL WALL PAIN}

Chronic pain caused by the abdominal wall may be often indistinguishable from internal organ pain. It may be associated with muscle injury (i.e., rectus abdominis, pyramidalis, external oblique, transversus abdominis) or nerve damage (i.e., iliohypogastric, ilioinguinal, genitofemoral, lateral femoral cutaneous, pudendal). Anterior cutaneous nerve entrapment syndrome is also associated with chronic abdominal wall pain. Birth, pelvic surgery, and trauma are frequently blamed in its etiopathogenesis. Dyspareunia may be
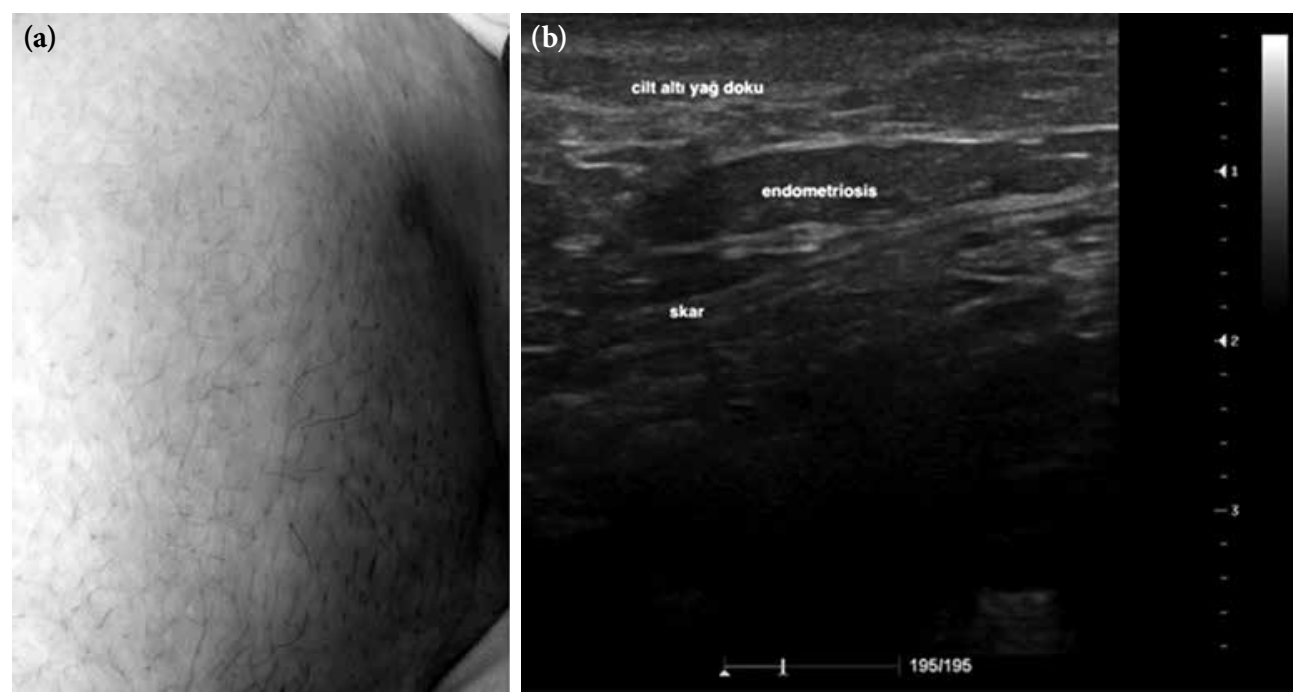

Figure 2. Anterior abdominal wall endometriosis. (a) A 43-year-old, multiparous woman presented with cyclic dull pain at the cesarean scar site for the past one year. (b) Ultrasonography revealed a subcutaneous nodule with irregular borders, a heterogeneous echo texture with endometriosis on left anterior abdominal wall. 
accompanied and sitting for extended periods may increase the pain. Postural disorders, abdominal muscles, thoracolumbar fascia, lumbar extensors, hip flexors, and abductor muscles balance disorders are often responsible for the development of chronic abdominal wall pain. ${ }^{[22]}$ Localized pain, a Carnett's sign, anesthetic injections may be helpful for the diagnosis. Patients are also advised to avoid intensive exercises for the abdominal muscles. ${ }^{[22,26-28]}$

\section{NEUROPELVEOLOGY}

Neuropelveology is an emerging discipline that focuses on the treatment of pelvic nerve disorders such as CPP, bladder and sexual dysfunction. ${ }^{[29]}$ In recent years, rehabilitation of pelvic nerve disorders after pelvic surgery in patients undergoing laparoscopic treatment of endometriosis, vaginal repair, and

Table 2. Common causes of groin pain

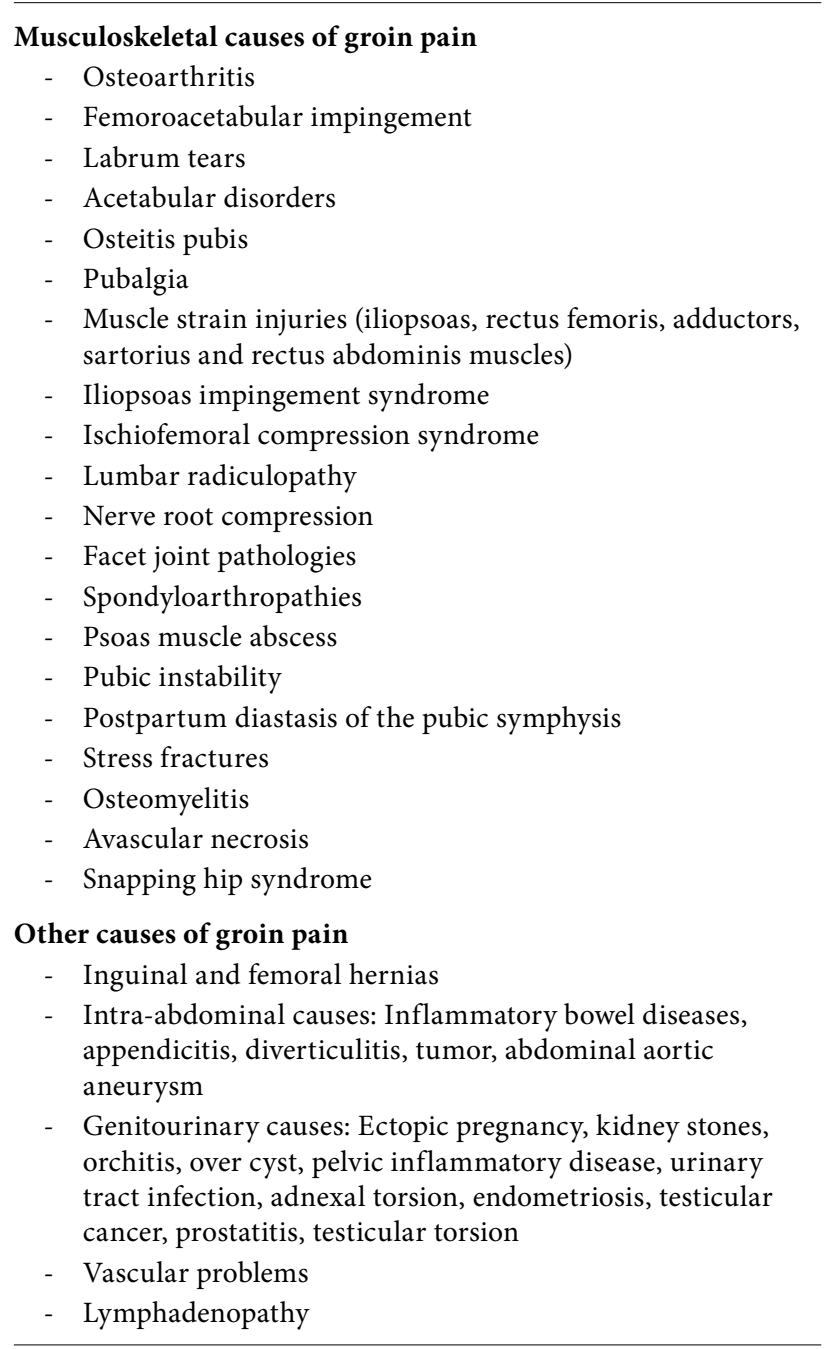

malignancy has been gaining importance in the management of pain and in the improvement of quality of life of patients. In a recent study, patients undergoing laparoscopic surgery for sciatic nerve endometriosis were evaluated and in patients with motor sciatica and pain complaints, followed by at least five years of follow-up with extensive resection of the sciatic nerve, a significant improvement in the physiological sciatic nerve function with improved walking was achieved with postoperative intensive physiotherapy and neuroleptic agents. ${ }^{[30]}$ The authors, therefore, placed a particular emphasis on the importance of intensive physiotherapy for the treatment success in the field of neuropelveology, particularly.

\section{TREATMENT AND REHABILITATION OF PELVIC PAIN}

The treatment and rehabilitation program can be planned to address specific causes and/or general pain treatment. Treatment should include a trial of conservative therapies (e.g. patient education, pharmacotherapy, psychotherapy, exercise, or physical therapy) that can often provide significant symptom relief and improved quality of life (Table 3).

Interventional and surgical methods can be used in conservative treatment-resistant cases. The methods such as ablative procedures, sacral neuromodulation, neurolysis, hysterectomy, vulvar, vestibular surgery, resection, and prostatectomy are applied..$^{[8]}$

\section{PATIENT EDUCATION, DIET MODIFICATION AND BEHAVIORAL THERAPY}

Behavioral therapy plays a critical role in the patient education. Timed urination, controlled fluid intake, provides symptomatic improvement in

Table 3. Conservative treatment of chronic pelvic pain

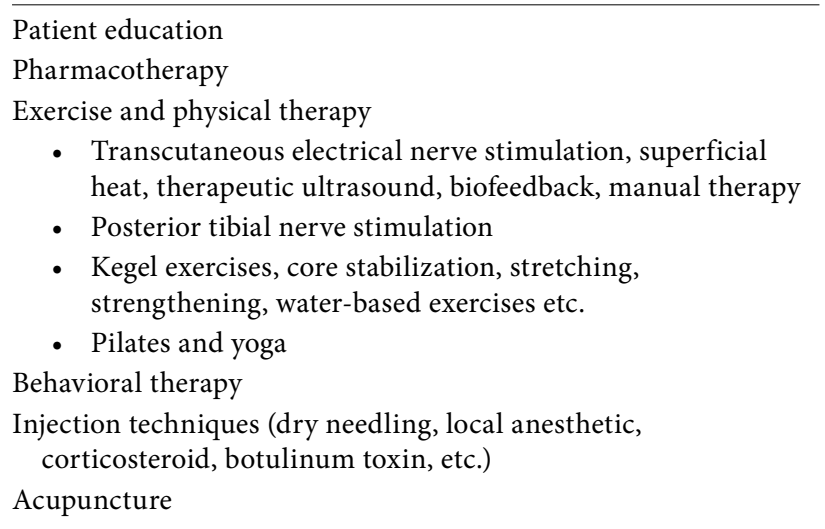


a significant proportion of patients with affected pelvic floor muscles. It is also possible to participate in stress coping strategies, patient school programs and support groups. Urogynecological disorders, particularly bladder diary and diet regimens, should be encouraged. Dietary modifications to prevent consumption of acidic drinks, coffee, tea, over-fat, spicy, gassy foods, large portions, alcohol and artificial sweeteners are very important in problems related to the gastrointestinal system, such as irritable bowel syndrome. Probiotics are recommended. ${ }^{[31]}$

\section{PHARMACOLOGICAL TREATMENT}

In patients with CPP, pharmacotherapy should be planned for the cause. Non-steroidal antiinflammatory drugs (NSAIDs), hormonal treatments, opioids, antidepressants, anticonvulsants, alpha blockers, and antibiotics are used in CPP, as indicated. Recognition and treatment of neuropathic pain in CPP are of utmost importance. In a study, 119 CPP patients were evaluated and anticonvulsants such as gabapentin and pregabalin were found to be effective in the treatment of $\mathrm{CPP}{ }^{[32]}$ Cheong et al. $^{[33]}$ reported that gabapentin was superior to amitriptyline. According to the results of the Cochrane database systematic review, pregabalin was not effective in patients with chronic prostatitis. ${ }^{[34]}$ However, further studies are needed to establish a definite conclusion. In addition, in a recent study, a comparative analysis of gene expression patterns from endometriosis and mouse models showed that the subset of $\alpha 2 \delta$ calcium channels in nociception might be the targets for the treatment of endometriosis-related pain. Also, small-scale clinical trials suggested that gabapentin could be effective in women with $\mathrm{CPP}{ }^{[35]}$

\section{PHYSIOTHERAPY AND REHABILITATION}

The efficacy of physiotherapy and rehabilitation applications in CPP treatment has been shown in several studies and guidelines. Individualized exercise programs as well as group exercises and initiatives to increase physical activity are important components of conservative treatment.

Methods such as exercise, manual therapy applications, biofeedback, transcutaneous electrical nerve stimulation, electrical stimulation, electromagnetic chair, extracorporeal shockwave therapy, acupuncture, and percutaneous tibial nerve neuromodulation are used in the treatment of CPP. Kegel exercises, posture, core stabilization, stretching, strengthening, flexibility, coordination exercises should be also included in the rehabilitation program (Figure 3). ${ }^{[3]}$ Of note, complex anatomy and overlapping pathologies make the diagnosis difficult in certain cases such as adductor muscle strain, iliopsoas syndrome, and gluteal tendinopathy. Therefore, comprehensive risk factors should be defined, all kinetic chains should be assessed, and the exercise program should be planned accordingly. ${ }^{[37]} \mathrm{In}$ a study conducted with transvaginal physiotherapy in patients with CPP, a significant decrease was observed in pain complaints of $63 \%$ of patients. ${ }^{[38]}$ In another multi-center study, patients with bladder pain syndrome/interstitial cystitis underwent physical therapy including manual therapy and exercise for the pelvic floor and hip circumference muscles, and a significant improvement was observed compared to those treated with global therapeutic massage. ${ }^{[39]}$ It has been proposed that manual therapy applied to the pelvic floor for 8 to 12 weeks, two days a week, decreases the pelvic floor hypertonic disorders and significantly improves the symptoms. ${ }^{[40]}$ According to the recently published Cochrane database systematic review, lifestyle modifications, physical activity, and acupuncture had positive effects in chronic prostatitis, while electromagnetic chair therapy was inadequate for the patients with chronic prostatitis. ${ }^{[41]}$ In addition, acupuncture and extracorporeal shockwave therapy had positive effects on chronic prostatitis symptoms and pain. ${ }^{[41]}$ Another study demonstrated that pain complaints of patients with chronic prostatitis/CPP significantly reduced with electroacupuncture. ${ }^{[42]}$

Istek et al. ${ }^{[43]}$ studied 33 women with CPP randomly assigned to either percutaneous tibial nerve stimulation (PTNS) or control. A total of 30-min PTNS for three days per week were applied for 12 weeks. Compared to the control group, a statistically significant improvement in pain and quality of life was achieved at Week 12. Also, improvement in the quality of life sustained for the PTNS group at six months. The authors suggested that PTSN treatment was a useful method in CPP treatment.

In a meta-analysis, patients with chronic prostatitis/CPP were found to benefit from the PTNS treatment, and a statistically significant improvement was observed in pain scores. ${ }^{[44]}$ Furthermore, it was shown that interventional sacral neuromodulation was effective in the treatment of patients affected by CPP, suggesting that this effect sustained over time. ${ }^{[45]}$ However, there is a limited number of studies comparing the efficacy of PTNS and sacral neuromodulation in the literature. 

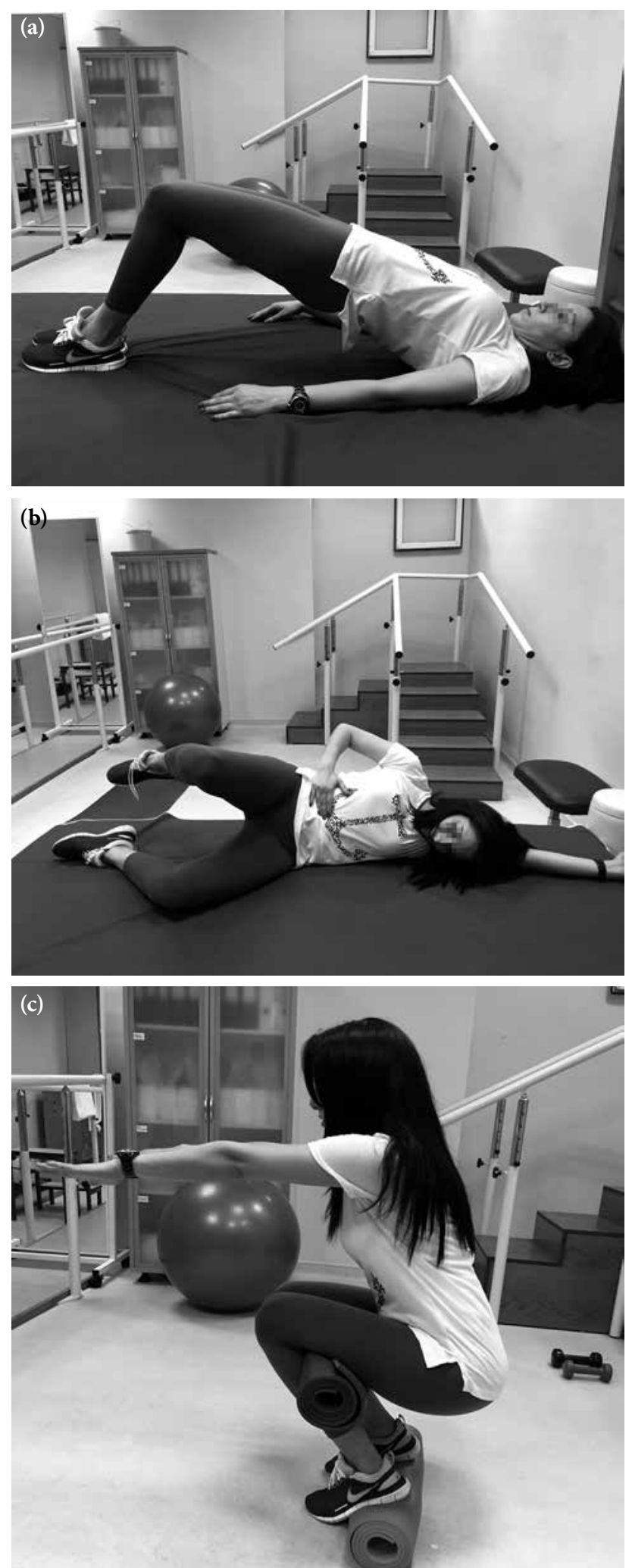

Figure 3. Therapeutic exercises for pelvic pain. (a) Abdominal and hip muscles strengthening exercises, bridge exercises. (b) Pelvic floor muscle exercises with diaphragmatic breathing. (c) Pelvic floor muscle and lower extremity strengthening exercises. ${ }^{[36]}$

\section{INJECTION TECHNIQUES IN PELVIC PAIN}

The pelvic region has many pain-producing regions, including certain specific parts where the joints, muscles, and nerve structures would be compressed. The influence of this compression may be accompanied by pelvic or back pain referred to the lower extremities. For the fields that cannot be visualized with USG, injections can be performed with the guidance of CT. The USG-guided injections and movement of real-time needle provides visualization advantage. Thus, important structures around the anatomical structures are avoided. ${ }^{[46]}$

Myofascial trigger points and ligaments, sacroiliac joint, facet joint, epidural injections and caudal epidural infiltration are performed in the pelvic region. It has been shown that myofascial pelvic pain contributes to 14 to $78 \%$ of the incidence of CPP. Dry needling, local anesthetics, saline and botulinum toxin injections can be applied. ${ }^{[8]}$ Trauma, muscle hypertrophy, inflammation, or nerve compromise from a neoplastic condition can be encountered as sciatalgia in piriformis syndrome. However, in rare cases, anatomic variations may be a source of refractory sciatalgia. ${ }^{[4]}$ Injection applications for piriformis muscle can be performed with the USG guidance. In patients with myofascial pelvic pain, there are studies of botulinum toxin administration to the resistant trigger points. A significant improvement was observed in $58 \%$ of patients with myofascial pelvic pain at eight weeks after the application of botulinum toxin to the iliococcygeus, puborectalis, obturator internus, and rectus muscles with physiotherapy program, including soft tissue release with transvaginal massage and stretching under anesthesia; however, no improvement was found in the patients with chronic bowel dysfunction in this study. In addition, a combination of botulinum toxin and physiotherapy for the management of pelvic myofascial pain is effective and patient selection is critical. ${ }^{[48]}$

The effectiveness of therapeutic nerve blocks performed with the guidance of USG and CT in CPP treatment has been shown in several studies. ${ }^{[4]}$ Nerve blocks are well-defined for acute pain; in addition, it is an active research field yielding positive results in recent studies. Ilioinguinal, iliohypogastric, genitofemoral blocks are used in the treatment of CPP. In particular, postoperative pain and perineal neuralgia applications are available. Applications for pudendal nerve can be applied in the treatment of endometriosis, pelvic adhesions, pudendal neuralgia, and chronic prostatitis. In addition, injection of transsacral block 
and lateral branches of posterior sacral roots can be performed. It is suggested that caudal blocks may be effective in interstitial cystitis. In addition, the ganglion impar block can be applied for pelvic cancer pain, coccydynia, and perineal pain conditions. ${ }^{[8,46,50,51]}$ Intravesical injection of local anesthetics, botulinum toxin and hyaluronic acid may be used in the treatment of refractory bladder pain syndrome. ${ }^{[52]}$

According to the European Association of Urology Guideline, the interdisciplinary collaboration in CPP management is particularly emphasized. Physiatrists, urologists, gynecologists, gastroenterologists, general surgeons, neurologists, psychiatrists, pain medicine physicians, physiotherapists, psychologists, and social workers are all involved in managing for CPP patients. This approach should combine the elements of medical, psychosocial and sexual care to bring the patient into a collaborative journey toward self-management. ${ }^{[53,54]}$

In conclusion, CPP is a complex and multifaceted disorder with the involvement of many organs including the urological, gynecological, musculoskeletal, and gastrointestinal systems. In addition, the treatment requires an integrated approach in which simultaneous consideration is paid to somatic, psychological, and social aspects. Conservative management gives satisfactory results in a high proportion of patients with CPP and, if conservative management fails, interventional approaches and surgery should be considered. Nonetheless, prospective, populationbased studies clearly are needed to establish the conditions in which abnormal musculoskeletal findings influence the development and management of CPP. A holistic approach should be preferred, with an emphasis to relieve the symptoms and to improve the quality of life of the patient. Involvement of the interdisciplinary team is central to the success and should be cooperated, where possible.

\section{Declaration of conflicting interests}

The authors declared no conflicts of interest with respect to the authorship and/or publication of this article.

\section{Funding}

The authors received no financial support for the research and/or authorship of this article.

\section{REFERENCES}

1. Le PU, Fitzgerald CM. Pelvic Pain: An Overview. Phys Med Rehabil Clin N Am 2017;28:449-54.

2. Bishop LA. Management of Chronic Pelvic Pain. Clin Obstet Gynecol 2017;60:524-30.

3. Juhan V. Chronic pelvic pain: An imaging approach. Diagn Interv Imaging 2015;96:997-1007.
4. Ahangari A. Prevalence of chronic pelvic pain among women: an updated review. Pain Physician 2014;17:141-7.

5. Bradley $\mathrm{MH}$, Rawlins A, Brinker CA. Physical Therapy Treatment of Pelvic Pain. Phys Med Rehabil Clin N Am 2017;28:589-601.

6. Weissman E, Boothe E, Wadhwa V, Scott K, Chhabra A. Magnetic Resonance Neurography of the Pelvic Nerves. Semin Ultrasound CT MR 2017;38:269-78.

7. Bharucha AE, Lee TH. Anorectal and Pelvic Pain. Mayo Clin Proc 2016;91:1471-86.

8. Gritsenko K, Cohen MS. Pelvik pain: In: Hurley RW, editor. Essentials of Pain Medicine. Philadelphia: Elsevier; 2017. p. 261-72.

9. Brawn J, Morotti M, Zondervan KT, Becker CM, Vincent K. Central changes associated with chronic pelvic pain and endometriosis. Hum Reprod Update 2014;20:737-47.

10. Riegel B, Bruenahl CA, Ahyai S, Bingel U, Fisch M, Löwe B. Assessing psychological factors, social aspects and psychiatric co-morbidity associated with Chronic Prostatitis/Chronic Pelvic Pain Syndrome (CP/CPPS) in men -- a systematic review. J Psychosom Res 2014;77:333-50.

11. Meister MR, Shivakumar N, Sutcliffe S, Spitznagle T, Lowder JL. Physical examination techniques for the assessment of pelvic floor myofascial pain: a systematic review. Am J Obstet Gynecol 2018. pii: S0002-9378(18)30530-1.

12. Temme KE, Pan J. Musculoskeletal Approach to Pelvic Pain. Phys Med Rehabil Clin N Am 2017;28:517-37.

13. Allaire C, Williams C, Bodmer-Roy S, Zhu S, Arion K, Ambacher K, et al. Chronic pelvic pain in an interdisciplinary setting: 1-year prospective cohort. Am J Obstet Gynecol 2018;218:114.e1-114.e12.

14. Moldwin RM, Fariello JY. Myofascial trigger points of the pelvic floor: associations with urological pain syndromes and treatment strategies including injection therapy. Curr Urol Rep 2013;14:409-17.

15. Sanses TV, Chelimsky G, McCabe NP, Zolnoun D, Janata J, Elston R, et al. The Pelvis and Beyond: Musculoskeletal Tender Points in Women With Chronic Pelvic Pain. Clin J Pain 2016;32:659-65.

16. FitzGerald MP, Kotarinos R. Rehabilitation of the short pelvic floor. I: Background and patient evaluation. Int Urogynecol J Pelvic Floor Dysfunct 2003;14:261-8.

17. Prendergast SA. Pelvic Floor Physical Therapy for Vulvodynia: A Clinician's Guide. Obstet Gynecol Clin North Am 2017;44:509-22.

18. Goldstein AT, Pukall CF, Brown C, Bergeron S, Stein A, Kellogg-Spadt S. Vulvodynia: Assessment and Treatment. J Sex Med 2016;13:572-90.

19. Khatri G, Khan A, Raval G, Chhabra A. Diagnostic Evaluation of Chronic Pelvic Pain. Phys Med Rehabil Clin N Am 2017;28:477-500.

20. Gambone JC, Mittman BS, Munro MG, Scialli AR, Winkel CA. Consensus statement for the management of chronic pelvic pain and endometriosis: proceedings of an expert-panel consensus process. Fertil Steril 2002;78:961-72.

21. Cody RF Jr, Ascher SM. Diagnostic value of radiological tests in chronic pelvic pain. Baillieres Best Pract Res Clin Obstet Gynaecol 2000;14:433-66. 
22. Koop H, Koprdova S, Schürmann C. Chronic Abdominal Wall Pain. Dtsch Arztebl Int 2016;113:51-7.

23. Devin CJ, McCullough KA, Morris BJ, Yates AJ, Kang JD. Hipspine syndrome. J Am Acad Orthop Surg 2012;20:434-42.

24. Dommerholt J, Finnegan M, Hooks T, Chou LW. A critical overview of the current myofascial pain literature - July 2018. J Bodyw Mov Ther 2018;22:673-84.

25. Bennis S, Hwang S. Office Evaluation of Pelvic Pain. Phys Med Rehabil Clin N Am 2017;28:461-76.

26. Oor JE, Ünlü Ç, Hazebroek EJ. A systematic review of the treatment for abdominal cutaneous nerve entrapment syndrome. Am J Surg 2016;212:165-74.

27. van Assen T, Boelens OB, van Eerten PV, Perquin C, Scheltinga $\mathrm{MR}$, Roumen RM. Long-term success rates after an anterior neurectomy in patients with an abdominal cutaneous nerve entrapment syndrome. Surgery 2015;157:137-43.

28. Mui J, Allaire C, Williams C, Yong PJ. Abdominal Wall Pain in Women With Chronic Pelvic Pain. J Obstet Gynaecol Can 2016;38:154-9.

29. Possover M. Laparoscopic management of neural pelvic pain in women secondary to pelvic surgery. Fertil Steril 2009;91:2720-5.

30. Possover M. Five-Year Follow-Up After Laparoscopic Large Nerve Resection for Deep Infiltrating Sciatic Nerve Endometriosis. J Minim Invasive Gynecol 2017;24:822-6.

31. Bharucha AE, Chakraborty S, Sletten CD. Common Functional Gastroenterological Disorders Associated With Abdominal Pain. Mayo Clin Proc 2016;91:1118-32.

32. Agarwal MM, Elsi Sy M. Gabapentenoids in pain management in urological chronic pelvic pain syndrome: Gabapentin or pregabalin? Neurourol Urodyn 2017;36:2028-33.

33. Cheong YC, Smotra G, Williams AC. Non-surgical interventions for the management of chronic pelvic pain. Cochrane Database Syst Rev 2014;3:CD008797.

34. Aboumarzouk OM, Nelson RL. Pregabalin for chronic prostatitis. Cochrane Database Syst Rev 2012;8:CD009063.

35. Bellessort B, Bachelot A, Grouthier V, De Lombares C, Narboux-Neme N, Garagnani P, et al. Comparative analysis of molecular signatures suggests the use of gabapentin for the management of endometriosis-associated pain. J Pain Res 2018;11:715-25.

36. Vural M. Role of birth in pelvicfloor dysfunction, postpartum exercises and Kegel exercises. In: Karan A, editor. Physical Therapy and Rehabilitation in Urogynecology. İstanbul: Nobel Tip Kitabevleri; 2016. s. 127-47.

37. Tyler TF, Fukunaga T, Gellert J. Rehabilitation of soft tissue injuries of the hip and pelvis. Int J Sports Phys Ther 2014;9:785-97.

38. Bedaiwy MA, Patterson B, Mahajan S. Prevalence of myofascial chronic pelvic pain and the effectiveness of pelvic floor physical therapy. J Reprod Med 2013;58:504-10.

39. FitzGerald MP, Payne CK, Lukacz ES, Yang CC, Peters KM, Chai TC, et al. Randomized multicenter clinical trial of myofascial physical therapy in women with interstitial cystitis/painful bladder syndrome and pelvic floor tenderness. J Urol 2012;187:2113-8.

40. Weiss JM. Pelvic floor myofascial trigger points: manual therapy for interstitial cystitis and the urgency-frequency syndrome. J Urol 2001;166:2226-31.

41. Franco JVA, Turk T, Jung JH, Xiao YT, Iakhno S, Garrote $\mathrm{V}$, et al. Non-pharmacological interventions for treating chronic prostatitis/chronic pelvic pain syndrome: a Cochrane systematic review. BJU Int 2018 Jul 18. [Epub ahead of print]

42. Lee SH, Lee BC. Electroacupuncture relieves pain in men with chronic prostatitis/chronic pelvic pain syndrome: three-arm randomized trial. Urology 2009;73:1036-41.

43. Istek A, Gungor Ugurlucan F, Yasa C, Gokyildiz S, Yalcin O. Randomized trial of long-term effects of percutaneous tibial nerve stimulation on chronic pelvic pain. Arch Gynecol Obstet 2014;290:291-8.

44. Cohen JM, Fagin AP, Hariton E, Niska JR, Pierce MW, Kuriyama A, et al. Therapeutic intervention for chronic prostatitis/chronic pelvic pain syndrome (CP/CPPS): a systematic review and meta-analysis. PLoS One 2012;7:e41941.

45. Martellucci J, Naldini G, Carriero A. Sacral nerve modulation in the treatment of chronic pelvic pain. Int $\mathrm{J}$ Colorectal Dis 2012;27:921-6.

46. Domingo-Rufes T, Bong DA, Mayoral V, Ortega-Romero A, Miguel-Pérez M, Sabaté A. Ultrasound-guided pain interventions in the pelvis and the sacral spine. Tech Reg Anesth Pain Manag 2013;17:107-30.

47. Ro TH, Edmonds L. Diagnosis and Management of Piriformis Syndrome: A Rare Anatomic Variant Analyzed by Magnetic Resonance Imaging. J Clin Imaging Sci 2018;8:6.

48. Halder GE, Scott L, Wyman A, Mora N, Miladinovic B, Bassaly R, et al. Botox combined with myofascial release physical therapy as a treatment for myofascial pelvic pain. Investig Clin Urol 2017;58:134-9.

49. Elkins N, Hunt J, Scott KM. Neurogenic Pelvic Pain. Phys Med Rehabil Clin N Am 2017;28:551-69.

50. Nagpal AS, Moody EL. Interventional Management for Pelvic Pain. Phys Med Rehabil Clin N Am 2017;28:621-46.

51. Le Clerc QC, Riant T, Levesque A, Labat JJ, Ploteau S, Robert R, et al. Repeated Ganglion Impar Block in a Cohort of 83 Patients with Chronic Pelvic and Perineal Pain. Pain Physician 2017;20:823-8.

52. Dellis A, Papatsoris AG. Intravesical treatment of bladder pain syndrome/interstitial cystitis: from the conventional regimens to the novel botulinum toxin injections. Expert Opin Investig Drugs 2014;23:751-7.

53. Engeler DS, Baranowski AP, Dinis-Oliveira P, Elneil S, Hughes J, Messelink EJ, et al. The 2013 EAU guidelines on chronic pelvic pain: is management of chronic pelvic pain a habit, a philosophy, or a science? 10 years of development. Eur Urol 2013;64:431-9.

54. Bornstein J, Goldstein AT, Stockdale CK, Bergeron S, Pukall C, Zolnoun D, et al. 2015 ISSVD, ISSWSH, and IPPS Consensus Terminology and Classification of Persistent Vulvar Pain and Vulvodynia. J Low Genit Tract Dis 2016;20:126-30. 